\title{
Numerical solutions of Maxwell's equations in 3D in frequency domain with linear sheath boundary conditions
}

\author{
W. Tierens, ${ }^{1}$ G. Urbanczyk, ${ }^{2,3}$ L. Colas, ${ }^{3}$ and M. Usoltceva ${ }^{1}$ \\ 1) Max Planck Institute of Plasma Physics, D-85748 Garching, Germany ${ }^{\mathrm{a})}$ \\ ${ }^{2)}$ Institute of Plasma Physics, Chinese Academy of Sciences, 230031 Hefei, China \\ ${ }^{3)}$ CEA, IRFM, F-13108 Saint Paul-Lez-Durance, France
}

(Dated: 15 July 2019)

\begin{abstract}
In this paper we construct a numerical technique capable of solving Maxwell's equations in frequency domain, both in vacuum and in cold magnetized plasma, with a boundary condition that guarantees the existence of a potential associated with the radiofrequency electric fields tangential to certain surfaces. This potential is of interest to nonlinear sheath physics, since it enables the calculation of the time-dependent sheath current excited by a single-frequency electromagnetic wave, and thereby the associated DC sheath current and sheath potential.
\end{abstract}

\section{INTRODUCTION}

In plasmas in general, and tokamak plasmas in particular, thermal sheaths form on all conductors in contact with the plasma. In the presence of radiofrequency (RF) waves, such as the ICRF (Ion Cyclotron Range of Frequencies) waves used to heat the plasma, the sheaths are excited. The potential drop across an excited sheath may be much larger than that across a standard Bohm sheath. It is this potential drop which accelerates ions towards the surfaces of the plasma-facing components, and in particular towards the surfaces of the ICRF antennas themselves.

Great progress has been made towards understanding and suppressing this phenomenon ${ }^{1,2}$, but a key ingredient for modelling sheaths in frequency domain in $3 \mathrm{D}$ is still missing: a potential needs to be associated with the radiofrequency electric fields tangential to the plasma-facing components. In 3D, it is hard to guarantee that this potential even exists.

In this paper, we develop a numerical technique to solve Maxwell's equations in 3D in cold magnetized plasma in frequency domain in a way that guarantees the existence of this RF potential. In section II, we briefly summarize the "SSWICH" approach to sheath modelling, and the sheath boundary conditions used therein. We relate these sheath boundary conditions to other types of sheath boundary conditions in section III. In section IV, we elucidate how the normal electric field components must constrain the tangential electric field components. Then, in section $\mathrm{V}$, we construct a numerical scheme capable of solving Maxwell's equations with these boundary conditions, the main novelty of this paper. To validate our numerical approach, we construct an exact solution in section VI. Finally, in section VII, we compare our numerical results with analytical results. The conclusion is in section VIII.

Throughout this paper, we use the subscripts $t$ and $n$ for tangential resp. normal quantities and operators. When more concreteness is needed, we use $x$ as the normal direction and $y, z$ as the tangential directions. We use a double arrow for tensors such as $\stackrel{\leftrightarrow}{\varepsilon}$, and a single arrow for vectors such as $\vec{E}$.

\footnotetext{
a) Electronic mail: wtt@ipp.mpg.de
}

\section{THE SSWICH APPROACH}

Within the "SSWICH" (Self-Consistent Sheaths and Waves for Ion Cyclotron Heating, ${ }^{1-3}$ ) approach, numerical modelling of sheath excitation on ICRF antennas typically follows a 3step process: Solving Maxwell's equations (section II A), calculating the RF potential (section IIB), and calculating the DC potential and sheath thickness (section II C).

\section{A. Step 1: Solving Maxwell's equations}

Solve Maxwell's equations in frequency domain in cold magnetized plasma near the antenna

$$
\nabla \times(\nabla \times \vec{E})+\frac{\omega^{2}}{c^{2}} \stackrel{\leftrightarrow}{\varepsilon} \vec{E}=i \omega \mu_{0} \vec{J}_{\mathrm{ant}}
$$

where $\vec{E}$ are the unknown electric fields near the antenna, $\omega$ is the angular antenna frequency, $\stackrel{\leftrightarrow}{\varepsilon}$ is the cold plasma dielectric tensor given in eqs. $(15,16)$. Near any surface, we can decompose $\vec{E}$ into a tangential component $\vec{E}_{t}$ and a normal component $\vec{E}_{n}$

$$
\vec{E}=\vec{E}_{t}+\vec{E}_{n}
$$

We typically solve eq. (1) with perfectly conducting boundary conditions on the metallic surfaces (PEC, $\vec{E}_{t}=0$ ), and perfectly matched layers (a type of absorbing boundary layer ${ }^{4}$ ) in the plasma. In this paper we will consider dedicated sheath boundary conditions: eqs. $(10,11,12)$.

\section{B. Step 2: Calculating RF potential}

To find the RF potential $\Psi_{R F}$ on a sheath surface, we need to solve $\mathrm{e}^{3,5-7}$

$$
-\nabla_{t} \Psi_{R F}=\vec{E}_{t}
$$

The conditions under which eq. (3) can be solved will be discussed in section II D. 


\section{Step 3: Calculating the DC potential}

$\Psi_{R F}$ rectifies the DC $I-V$ characteristic of the sheath ${ }^{1}$ as follows:

$$
I_{D C}=j^{+}\left(1-\exp \left(\frac{e}{k_{B} T_{e}}\left(V_{f}+V_{b}-V_{D C}\right)\right)\right)
$$

where the ion saturation current $j^{+}$, the floating potential $V_{f}$, and the biasing voltage $V_{b}$ are respectively

$$
\begin{aligned}
j^{+} & =\frac{1}{2} e n_{i} c_{s} \\
V_{f} & =\frac{k_{B} T_{e}}{2 e} \ln \left(2 \pi \frac{m_{e}}{m_{i}}\left(1+\frac{T_{i}}{T_{e}}\right)\right) \\
V_{b} & =\frac{k_{B} T_{e}}{e} \ln \left(I_{0}\left(\frac{e \Psi_{R F}}{k_{B} T_{e}}\right)\right)
\end{aligned}
$$

where $I_{0}$ is the modified Bessel function of order $0, n_{i}$ the ion density, $c_{s}$ the speed of sound, $T_{e}, T_{i}$ the electron and ion temperature. Eq. (7) is an approximation due to the twofrequency approach of SSWICH: only the antenna frequency and the DC frequency are considered, and other harmonics which $V_{b}$ contains are ignored.

We solve the continuity equation for DC currents in the scrape-off-layer, in presence of anisotropic DC conductivity $\stackrel{\leftrightarrow}{\sigma}$ in the plasma volume

$$
\nabla \cdot \vec{J}_{D C}=-\nabla \cdot \stackrel{\leftrightarrow}{\sigma} \nabla V_{D C}=0
$$

using eq. (4) as boundary condition. This gives the DC sheath potential $V_{D C}$, and the sheath width $\delta_{s h}$ via the ChildLangmuir law:

$$
\delta_{s h}=\lambda_{D e}\left(\frac{e V_{D C}}{k_{B} T_{e}}\right)^{3 / 4}
$$

where $\lambda_{D e}$ is the electron Debeye length.

\section{Requirements for RF potential calculation}

In this work, we are concerned with steps 1 and 2, and specifically, with the boundary conditions that we must impose while solving Maxwell's equations (step 1) to guarantee that an RF potential can be calculated (step 2). The goal is to solve these steps together, provided that the sheath width $\delta_{s h}$ is prescribed a priori. The sheath width can then be selfconsistently determined as in figure 1 .

Eq. (3) states that the RF electric fields tangential to the sheath surface are gradients of the RF potential $\Psi_{R F}$, a scalar function on that surface. Under which conditions can a vector field $\vec{E}_{t}$ tangent to a surface be the tangential gradient of a potential $\Psi_{R F}$ defined on that surface? The vector field must be conservative: $(\nabla \times \vec{E})_{n}=0$. In the context of time-harmonic electrodynamics, this is strictly equivalent to $B_{n}=0$ all over the boundary. For 2D problems (invariant in the out-of-plane direction) $B_{n}=0$ is automatically fulfilled. To complement the boundary conditions, one needs another scalar equation defined on the boundary and describing the sheath properties at the RF level (typically the RF impedance of the sheath). This is the role of equation (11). Eqs. (10) and (11) are the "asymptotic linear sheath boundary conditions" $(\mathrm{SBC})^{3}$

$$
\begin{aligned}
(\nabla \times \vec{E})_{n} & =0 \text { or equivalently } \nabla_{t} \times \vec{E}=0 \\
D_{n} & =0
\end{aligned}
$$

If the sheath thickness $\delta_{s h}$ is known, we want to impose the "non-asymptotic sheath boundary condition" instead ${ }^{3,8}$ :

$$
\left\{\begin{array}{ccc}
\nabla_{t} \times \vec{E}_{t} & = & 0 \\
\Psi_{R F} & = & -\frac{\delta_{s h}}{\varepsilon_{s h}} D_{n}
\end{array}\right.
$$

where $\varepsilon_{s h}$ an effective scalar dielectric constant of order 1 for capacitive sheaths. $\varepsilon_{s h}=1$ represents a capacitive limit in which there is no power dissipation in the sheath, but in general there is also a resistive term ${ }^{5}$, and $\varepsilon_{s h}$ may be complex. The asymptotic sheath boundary conditions are a limiting case of the non-asymptotic sheath boundary conditions ${ }^{9}$ as $\delta_{s h} \rightarrow \infty$.

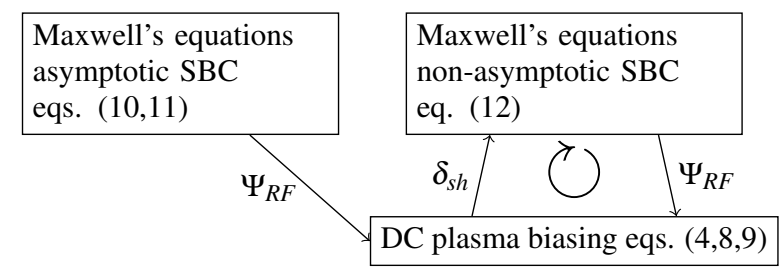

FIG. 1. Given the ability to solve Maxwell's equations with asymptotic SBCs (Eqs. 10,11) and non-asymptotic SBCs (eq. 12), the sheath thickness $\delta_{s h}$ can be determined self-consistently by repeatedly iterating the DC biasing step (section IIC), which determines $\delta_{s h}$ given $\Psi_{R F}$, and solving Maxwell's equations with nonasymptotic SBCs, which determines $\Psi_{R F}$ given $\delta_{s h}$.

Although eqs. (10) and (11) suffice to uniquely (and nontrivially) define $\Psi_{R F}$, implementing these boundary conditions within commercial Finite Element solvers has proven challenging $^{3}$. So far, SSWICH has gotten around this problem by resorting to $2 \mathrm{D}$ approaches ${ }^{1-3}$ : in a $2 \mathrm{D}$ domain, eq. (3) reduces to a $1 \mathrm{D}$ problem, and in $1 \mathrm{D}$ all vector fields are conservative. Among the more sophisticated 2D approaches is the "multi-2D" or "multislice" approach ${ }^{1-3,10}$ (figure 2), in which the antenna is sliced up into multiple 2D radial-parallel slices. The $2 \mathrm{D}$ problem is solved on each slice, and an attempt is made to reconstruct $3 \mathrm{D}$ information by stitching the slices together. Another possibility to take into account the 3D nature of the original problem is to use a 2D slice with a Fourier expansion in the vertical (poloidal) direction ${ }^{9}$. Both of these approaches require the geometry to be invariant in that direction, which prevents us from taking into account the effect of the Faraday screen, or study sheath formation on the upper and lower limiters. 


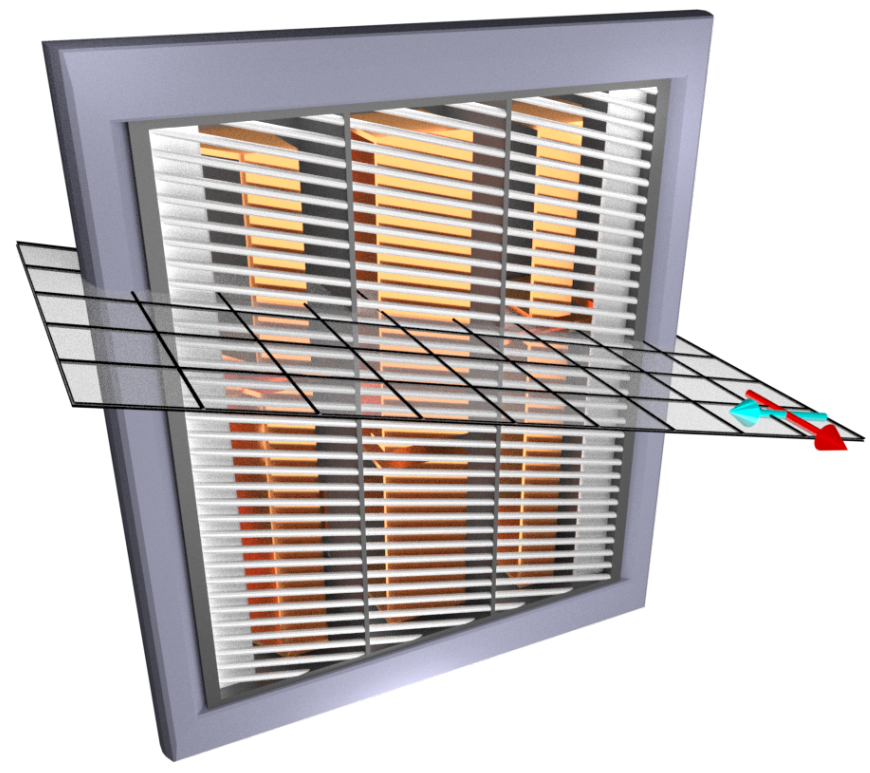

FIG. 2. In the multi-2D approach, $\Psi_{R F}$ is calculated on many 2D radial-parallel slices (radial: red arrow, parallel (to the confining magnetic field): cyan arrow). One such slice is shown here with the flat RAPLICASOL model ${ }^{2,11,12}$ of the ASDEX Upgrade 3-strap antenna. On every slice, eq. (3) reduces to a $1 \mathrm{D}$ problem so it can be solved even if the $2 \mathrm{D}$ tangential fields are non-conservative.

\section{SHEATH BOUNDARY CONDITIONS IN TIME AND FREQUENCY DOMAIN}

Jenkins and Smithe ${ }^{13-15}$ propose the following timedomain sheath boundary condition. Ignoring loss terms, terms related to dielectric coatings, and assuming known constant sheath width $\delta_{s h}$ :

$$
\frac{\partial \phi_{R F}}{\partial t}=-\frac{\delta_{s h}}{\varepsilon_{0}} \overrightarrow{1}_{n} \cdot \sum_{s \in \text { species }} \vec{J}_{s}(t)
$$

where $\overrightarrow{1}_{n}$ is the unit vector normal to the surface. We use $\phi_{R F}$ instead of $\Psi_{R F}$ for reasons that will become clear shortly. Eq. (13) states that the sheath is excited by the normal component of the currents of the plasma species that enter the sheath.

$\phi_{R F}$ is then coupled to Maxwell's equations not via the tangential fields, but by including a sheath term in Faraday's law in integral form, wherever the integration path passes through a sheath. In fact, the total potential drop across the sheath is assumed to be

$$
\Psi_{R F}=\phi_{R F}-\delta_{s h} E_{n, 0}
$$

where $E_{n, 0}$ is the normal component of the electric field just outside the sheath (so $\phi_{R F}$ gives the potential drop "on top of" $\left.E_{n, 0}\right)$. The $\delta_{s h} E_{n, 0}$ term accounts for displacement currents, the other term $\phi_{R F}$ is given entirely by particle currents.

In cold magnetized plasma, the dielectric tensor is Hermi- tian, and can be written as a sum over the particle species ${ }^{16}$ :

$$
\begin{aligned}
& \stackrel{\leftrightarrow}{\varepsilon}=1+\sum_{s \in \text { species }} \overleftrightarrow{\varepsilon}_{s} \\
& \overleftrightarrow{\varepsilon}_{s}=-\left[\begin{array}{ccc}
\frac{\omega_{p, s}^{2}}{\omega^{2}} & 0 & 0 \\
0 & \frac{\omega_{p, s}^{2}}{\omega^{2}-\Omega_{s}^{2}} & \frac{\Omega_{s}}{-i \omega} \frac{\omega_{p, s}^{2}}{\omega^{2}-\Omega_{s}^{2}} \\
0 & \frac{\Omega_{s}}{i \omega} \frac{\omega_{p, s}^{2}}{\omega^{2}-\Omega_{s}^{2}} & \frac{\omega_{p, s}^{2}}{\omega^{2}-\Omega_{s}^{2}}
\end{array}\right]
\end{aligned}
$$

The specific form of the dielectric tensor in eq. (16) is valid assuming the confining magnetic field $B$ is along the $x$ axis, but this is not a necessary assumption anywhere in this paper. $\omega$ is the (angular) antenna frequency, $\omega_{p, s}=\sqrt{\frac{n_{s} q_{s}^{2}}{m_{s} \varepsilon_{0}}}$ the plasma frequency of a given species $s$, and $\Omega_{s}=\frac{q_{s} B}{m_{s}}$ the cyclotron frequency of a given species $s$.

In frequency domain, the current associated with species $s$ is $^{17,18} \vec{J}_{s}=\varepsilon_{0} \stackrel{\leftrightarrow}{\varepsilon}_{s} i \omega \vec{E}$, so eq. (13) becomes

$$
\begin{aligned}
i \omega \phi_{R F} & =-\frac{\delta_{s h}}{\varepsilon_{0}} \overrightarrow{1}_{n} \cdot \sum_{s \in \text { species }} \varepsilon_{0} \overleftrightarrow{\varepsilon}_{s} i \omega \vec{E} \\
\phi_{R F} & =-\delta_{s h} \overrightarrow{1}_{n} \cdot \sum_{s \in \text { species }} \overleftrightarrow{\varepsilon}_{s} \vec{E} \\
\phi_{R F} & =-\delta_{s h} \overrightarrow{1}_{n} \cdot(\stackrel{\leftrightarrow}{\varepsilon}-1) \vec{E} \\
\phi_{R F}-\delta_{s h} E_{n, 0} & =-\delta_{s h} \overrightarrow{1}_{n} \cdot \stackrel{\leftrightarrow}{\varepsilon} \vec{E}
\end{aligned}
$$

We recognize eq. (14) in eq. (20)

$$
\begin{aligned}
\Psi_{R F} & =-\delta_{s h} \overrightarrow{1}_{n} \cdot \stackrel{\leftrightarrow}{\varepsilon} \vec{E} \\
& =-\delta_{s h} D_{n}
\end{aligned}
$$

which is the non-asymptotic sheath boundary condition eq. (12) assuming $\varepsilon_{\text {sh }}=1$.

We conclude that the time-domain sheath boundary conditions of ${ }^{13-15}$, when transformed to frequency domain, are nearly equivalent to the frequency-domain sheath boundary conditions considered here, with the sole difference being the value of $\varepsilon_{s h}$. They are exactly equivalent when $\varepsilon_{s h}=1$, i.e. for purely capacitive sheaths.

In line with the SSWICH approach to sheath modelling, this paper will consider ways to solve Maxwell's equations with this boundary condition directly in frequency domain.

\section{RELATION BETWEEN TANGENTIAL AND NORMAL ELECTRIC FIELD COMPONENTS IN PRESENCE OF SHEATH BOUNDARY CONDITIONS}

\section{A. Relation between tangential and normal electric field components for a SBC in vacuum}

The boundary conditions eqs. (10) and (11) are physically meaningful even in vacuum: they also describe the boundary conditions at an interface vacuum / thin dielectric medium / perfectly conducting ground plane. Then, $\Psi_{R F}$ is the potential across the dielectric layer. 
The electric fields at a sheath boundary in vacuum are determined by

$$
\left\{\begin{array}{ccccc}
\nabla_{t} \cdot \vec{E}_{t} & = & -\partial_{n} \vec{E}_{n} & \text { No net charges } & (a) \\
\nabla_{t} \times \vec{E}_{t} & = & 0 & \text { Conservative } \vec{E}_{t} & (b) \\
\vec{E}_{n} & = & 0 & \text { Asymptotic SBC } & (c)
\end{array}\right.
$$

Thus, the tangential electric fields have known curl and divergence, which suffices to uniquely specify them.

Inserting $\vec{E}_{t}=-\nabla_{t} \Psi_{R F}$ into eq. (22) turns eq. (22.a) into a Poisson equation, and (22.b) becomes trivially true. Thus, eq. (22) is equivalent to eq. (23):

$$
\left\{\begin{array}{ccccc}
\nabla_{t}^{2} \Psi_{R F} & = & \partial_{n} \vec{E}_{n} & \text { Poisson equation for } \Psi_{R F} & (a) \\
\vec{E}_{t} & = & -\nabla \Psi_{R F} & \text { Tangential } \vec{E} \text { is conservative } & (b) \\
\vec{E}_{n} & = & 0 & \text { Asymptotic SBC } & (c)
\end{array}\right.
$$

There are reasons to prefer the "Poisson formulation" eq. (23) over the "local formulation" eq. (22). First, the local formulation does not generalize to the non-asymptotic sheath bounrary condition eq. (12), which requires explicit knowledge of $\Psi_{R F}$ to constrain $D_{n}$. Second, we have found the Poisson formulation to be better conditioned numerically, which will be discussed in the numerical examples (figure 14).

\section{B. Relation between tangential and normal electric field components for a SBC in plasma}

Instead of $\nabla \cdot \vec{E}=0$ (absence of net charges in vacuum), in plasma we have $\nabla \cdot \vec{D}=\nabla \cdot \overleftrightarrow{\varepsilon} \vec{E}=0$ (conservation of displacement ${ }^{19,20} /$ microscopic current continuity). Thus, the boundary conditions analogous to the local formulation eq. (22) are

$$
\left\{\begin{array}{ccccc}
\nabla_{t} \cdot \vec{D}_{t} & = & -\partial_{n} \vec{D}_{n} & \text { Microscopic current continuity } & (a) \\
\nabla_{t} \times \vec{E}_{t} & = & 0 & \text { Tangential } \vec{E} \text { is conservative } & (b) \\
\vec{D}_{n} & = & 0 & \text { Asymptotic SBC } & (c)
\end{array}\right.
$$

It will be useful to have a plasma equivalent of the Poisson formulation, eq. (23). For this, we need an operator $\overleftrightarrow{\varepsilon}_{t}$ which maps the tangential electric field $\vec{E}_{t}=-\nabla_{t} \Psi_{R F}$ onto the tangential electric displacement field $\vec{D}_{t}$. In general, knowledge of $\vec{E}_{t}$ does not suffice to find $\vec{D}_{t}: \stackrel{\leftrightarrow}{\varepsilon}$ may be a general $3 \times 3$ matrix, and $\vec{D}_{t}$ may depend also on $E_{n}$. However, we can use the asymptotic SBC $D_{n}=0$ to obtain a unique mapping $\overleftrightarrow{\varepsilon}_{t}: \vec{E}_{t} \rightarrow \vec{D}_{t}$ as follows: Suppose the tangential directions are $y, z$, and the normal direction is $x$, then at the SBC we have

$$
\stackrel{\leftrightarrow}{\varepsilon}\left[\begin{array}{c}
E_{x} \\
E_{y} \\
E_{z}
\end{array}\right]=\left[\begin{array}{c}
0 \\
D_{y} \\
D_{z}
\end{array}\right]
$$

which can be solved for $D_{y}, D_{z}$ and $E_{x}$ in terms of $E_{y}, E_{z}$. This solution defines $\overleftrightarrow{\varepsilon}_{t}:\left(E_{y}, E_{z}\right) \rightarrow\left(D_{y}, D_{z}\right)$. Explicitly,

$$
\left[\begin{array}{c}
D_{y} \\
D_{z}
\end{array}\right]=\overleftrightarrow{\varepsilon}_{t}\left[\begin{array}{c}
E_{y} \\
E_{z}
\end{array}\right] \Longleftrightarrow\left\{\begin{aligned}
D_{y} & =-\frac{\left(\varepsilon_{12} \varepsilon_{21}-\varepsilon_{11} \varepsilon_{22}\right)}{\varepsilon_{11}} E_{y} \\
& -\frac{\left(\varepsilon_{13} \varepsilon_{21}-\varepsilon_{11} \varepsilon_{23}\right)}{\varepsilon_{11}} E_{z} \\
D_{z} & =-\frac{\left(\varepsilon_{12} \varepsilon_{31}-\varepsilon_{11} \varepsilon_{32}\right)}{\varepsilon_{11}} E_{y} \\
& -\frac{\left(\varepsilon_{13} \varepsilon_{31}-\varepsilon_{11} \varepsilon_{33}\right)}{\varepsilon_{11}} E_{z}
\end{aligned}\right.
$$

$\stackrel{\leftrightarrow}{\varepsilon}_{t}$ is Hermitian, like $\stackrel{\leftrightarrow}{\varepsilon}$ itself. If the confining magnetic field is perpendicular to the sheath surface, $\stackrel{\leftrightarrow}{\varepsilon}_{t}$ happens to equal a $2 \times 2$ sub-block of $\stackrel{\leftrightarrow}{\varepsilon}$, but that is not true for general magnetic field orientations.

Inserting $\vec{D}_{t}=-\stackrel{\leftrightarrow}{\varepsilon}_{t} \nabla \Psi_{R F}$ into eq. (24.a), we get the Poisson formulation

$$
\nabla_{t} \cdot \stackrel{\leftrightarrow}{\varepsilon}_{t} \nabla \Psi_{R F}=\partial_{n} \vec{D}_{n}
$$

\section{Non-asymptotic case}

Consider the linear operator $\left[\Psi_{R F} \rightarrow \vec{D}_{t}\right]$ which maps $\Psi_{R F}$ onto $\vec{D}_{t}$ :

$$
\vec{D}_{t}=\left[\Psi_{R F} \rightarrow \vec{D}_{t}\right] \Psi_{R F}
$$

In vacuum, $\left[\Psi_{R F} \rightarrow \vec{D}_{t}\right]=-\varepsilon_{0} \nabla$. In plasma with asymptotic SBC, $\left[\Psi_{R F} \rightarrow \vec{D}_{t}\right]=-\overleftrightarrow{\varepsilon}_{t} \nabla$. In the non-asymptotic case, $\vec{D}_{t}$ depends on $\Psi_{R F}$ itself, not just on its gradient. We can no longer factor $\left[\Psi_{R F} \rightarrow \vec{D}_{t}\right]$ as a product of tensor and the gradient operator, we can only define it by

$$
\vec{D}_{t}=\left[\Psi_{R F} \rightarrow \vec{D}_{t}\right] \Psi_{R F} \Longleftrightarrow \overleftrightarrow{\varepsilon}\left[\begin{array}{c}
E_{x} \\
-\partial_{y} \Psi_{R F} \\
-\partial_{z} \Psi_{R F}
\end{array}\right]=\left[\begin{array}{c}
-\frac{\varepsilon_{s h}}{\delta_{s h}} \Psi_{R F} \\
D_{y} \\
D_{z}
\end{array}\right]
$$

The Poisson-like equation at the non-asymptotic SBC, analogous to eqs. (23.a) and (27), is

$$
\nabla_{t} \cdot\left[\Psi_{R F} \rightarrow \vec{D}_{t}\right] \Psi_{R F}=\partial_{n} \vec{D}_{n}
$$

\section{Boundary conditions for $\Psi_{R F}$}

We have shown that $\Psi_{R F}$ can be found by solving the second-order partial differential equations (23.a),(27) or (30) for the vacuum, plasma, and non-asymptotic cases respectively. Still, $\Psi_{R F}$ should be defined by the first-order equation (3), and thus be defined up to a constant. This remains true even when $\Psi_{R F}$ is defined by one of the second-order partial differential equations, but the details depend on the topology of the surface on which $\Psi_{R F}$ is defined, as shown in figure 3 . 

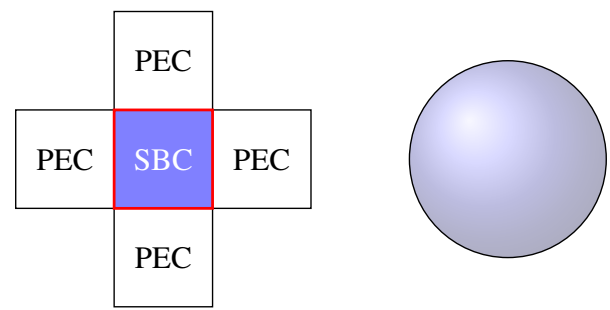

FIG. 3. Left: If a surface with sheath boundary condition (SBC, blue) is surrounded by surfaces with perfect electric conductor (PEC) boundary conditions, or other boundary conditions on which the tangential electric field is known, the integral of the known tangential electric field along the boundary of the SBC surface (red) gives, up to a constant, a Dirichlet boundary condition for $\Psi_{R F}$ on the sheath surface (blue). In the case of surrounding PEC boundary conditions, the Dirichlet boundary condition is that $\Psi_{R F}$ is constant along the boundary of the SBC surface (red), and this constant may be arbitrarily chosen to be 0 .

Right: If the surface with sheath boundary condition (SBC, blue) is freely floating in the plasma, such as this sphere, then the secondorder Poisson-like equations define $\Psi_{R F}$ up to a constant, due to the non-existence of nontrivial solutions to $\nabla^{2} f=0$ on such a domain ${ }^{21}$.

\section{A NUMERICAL APPROACH BASED ON FINITE DIFFERENCES}

\section{A. Configuration}

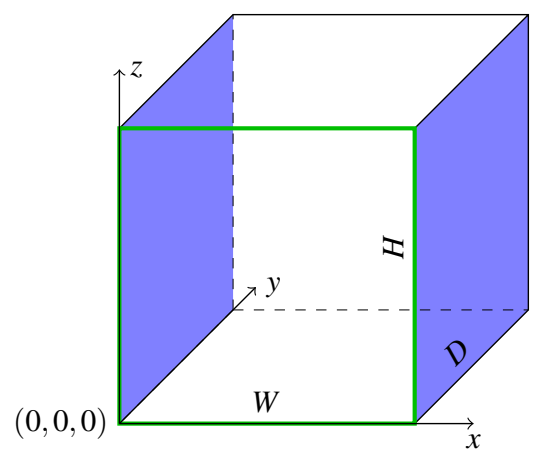

FIG. 4. Test geometry, with sheath surfaces in blue $(x=0, x=W)$ and the surface with source boundary condition outlined in green $(y=0)$. The three remaining surfaces have PEC boundary conditions.

We solve Maxwell's equations in a cuboid of size $W \times D \times$ $H$ as shown in figure 4. At $x=0, x=W$ we impose sheath boundary conditions. At $y=0$ we impose known tangential fields as a source condition. On the three remaining sides we impose PEC boundary conditions.

As discussed in section IV D, the PEC and source boundary conditions give us the tangential electric field along the edges of the sheath surfaces, which can be integrated to find $\Psi_{R F}$ along those edges, which gives us (up to an arbitrary constant) a Dirichlet boundary condition for $\Psi_{R F}$.

This configuration, though it is very much a test case to validate the numerics, is not as completely unrealistic as one might think: suppose the $y=0$ plane (green in figure 4) is the aperture of an ICRF antenna, just in front of the Faraday screen. If the waves decay quickly enough in the $y$ direction such that reflection at the $y=D$ plane can be neglected, $\Psi_{R F}$ on the $x=0$ and $x=W$ planes (blue in figure 4) is the RF potential on the antenna limiters.

\section{B. Discretisation}

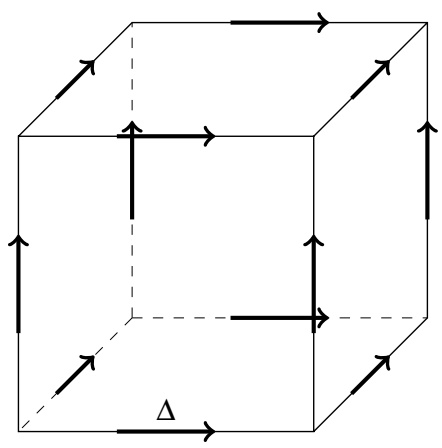

FIG. 5. The test geometry is discretized using Yee cells of side length $\Delta$, where the electric field components (black arrows) are associated with cell edges, and the magnetic field components (not shown) with cell faces.

We fill the cuboid with standard Yee cells (figure 5, see also $^{22}$ ) of size $\Delta \times \Delta \times \Delta$ (and let $W, D, H$ be integer multiples of $\Delta$ ). Suppose our vector of discretized electric resp. magnetic field components is $\mathscr{E}$ resp. $\mathscr{B}$, and the finite-difference curl operator is $C$. Then, the standard finite-difference discretisation of Maxwell's equations is

$$
\begin{aligned}
C \mathscr{E} & =-i \omega \mathscr{B} \\
C^{T} \mathscr{B} & =i \omega \varepsilon_{0} \mu_{0} \mathscr{E}
\end{aligned}
$$

We will denote by $\mathscr{E}_{x=q}$ the subset of discretized electric field components at $x=q$. By the Yee cell's construction, if $q$ is an integer multiple of $\Delta, \mathscr{E}_{x=q}$ only contains discrete $E_{y}$ and $E_{z}$ components (parallel to the sheath surfaces), and when $q$ is a half-integer multiple of $\Delta, \mathscr{E}_{x=q}$ only contains discrete $E_{x}$ components (normal to the sheath surfaces).

In what follows, we will require the solution of equations (23.a),(27) and (30) (with Dirichlet boundary conditions imposed by the neighbouring source and PEC planes as discussed in section IV D). With minor abuse of notation we will write

$$
\begin{gathered}
\nabla_{t}^{2} \Psi_{R F}=\partial_{n} \vec{E}_{n} \Longleftrightarrow \Psi_{R F}=\left(\nabla_{t}^{2}\right)^{-1} \partial_{n} \vec{E}_{n} \\
\nabla_{t} \cdot \overleftrightarrow{\varepsilon}_{t} \nabla_{t} \Psi_{R F}=\partial_{n} \vec{D}_{n} \Longleftrightarrow \Psi_{R F}=\left(\nabla_{t} \cdot \overleftrightarrow{\varepsilon}_{t} \nabla_{t}\right)^{-1} \partial_{n} \vec{D}_{n} \\
\nabla_{t} \cdot\left[\Psi_{R F} \rightarrow \vec{D}_{t}\right] \Psi_{R F}=\partial_{n} \vec{D}_{n} \Longleftrightarrow \\
\Psi_{R F}=\left(\nabla_{t} \cdot\left[\Psi_{R F} \rightarrow \vec{D}_{t}\right]\right)^{-1} \partial_{n} \vec{D}_{n}
\end{gathered}
$$


We will also assume that these solutions can be found on the discrete 2D Yee grid that is the sheath surface. The theory of solving Poisson-like equations on such grids is well-known and needs not be repeated here ${ }^{23,24}$.

\section{In vacuum}

We first consider the vacuum case. We need to solve

$$
\left\{\begin{array}{cccc}
C \mathscr{E} & = & -i \omega \mathscr{B} & \text { everywhere } \\
C^{T} \mathscr{B} & = & i \omega \varepsilon_{0} \mu_{0} \mathscr{E} & \text { not at SBC } \\
\mathscr{E}_{x=0}= & -\nabla_{t}\left(\nabla_{t}^{2}\right)^{-1} \frac{\mathscr{E}_{x=\Delta / 2}-0}{\Delta / 2} & \text { left SBC } \\
\mathscr{E}_{x=W}= & -\nabla_{t}\left(\nabla_{t}^{2}\right)^{-1} \frac{0-\mathscr{E}_{x=W-\Delta / 2}}{\Delta / 2} & \text { right SBC }
\end{array}\right.
$$

The discrete operator $\nabla_{t}\left(\nabla_{t}^{2}\right)^{-1}$, when expressed as a matrix, is not sparse. This is not a real problem (unless we insist on using direct solvers), since to solve eq. (36) iteratively, we merely need to be able to evaluate $\nabla_{t}\left(\nabla_{t}^{2}\right)^{-1}$ acting on some vector, we do not need its explicit matrix form.

If we do want to solve a sparse system of equations, we can equivalently solve

$$
\left\{\begin{array}{cccc}
C \mathscr{E} & = & -i \omega \mathscr{B} & \text { everywhere } \\
C^{T} \mathscr{B} & = & i \omega \varepsilon_{0} \mu_{0} \mathscr{E} & \text { not at SBC } \\
\nabla_{t} \cdot \mathscr{E}_{x=0} & = & -\frac{\mathscr{E}_{x=\Delta / 2}-0}{\Delta / 2} & \\
\nabla_{t} \times \mathscr{E}_{x=0} & = & 0 & \text { left SBC } \\
\nabla_{t} \cdot \mathscr{E}_{x=W} & = & -\frac{0-\mathscr{E}_{x=W-\Delta / 2}}{\Delta / 2} & \text { right SBC } \\
\nabla_{t} \times \mathscr{E}_{x=W} & = & 0 & \text { right SBC }
\end{array}\right.
$$

This requires some care to make sure that the discrete curl and divergence equations at the sheaths add up to full-rank systems of equations of the desired size (figure 6), but is otherwise equivalent to eq. (36).

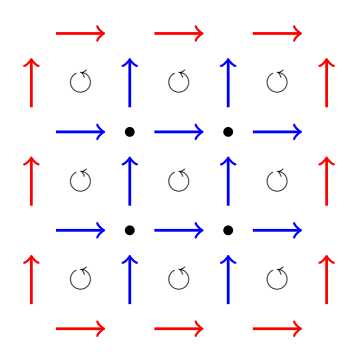

FIG. 6. Suppose this $3 \times 3$ Yee grid is the $x=0$ sheath surface. 12 degrees of freedom are not prescribed by other boundary conditions (blue arrows). There are 4 natural points to enforce $\nabla \cdot \vec{E}=0$ (black dots), and 9 natural points to enforce $\nabla_{t} \times \vec{E}_{t}=0$ (indicated by $\circlearrowleft$ ). Provided the other boundary conditions (red arrows) are compatible with the existence of a potential, that is, provided the red arrows integrate to 0 , the 9 curl equations have rank 8 , giving a total of 12 distinct equations to enforce on the surface - enough to fully specify the 12 tangential field unknowns (blue arrows). This observation generalizes to rectangular grids of arbitrary size.

\section{In plasma}

Analogous to eqs. (36) and (37), we have

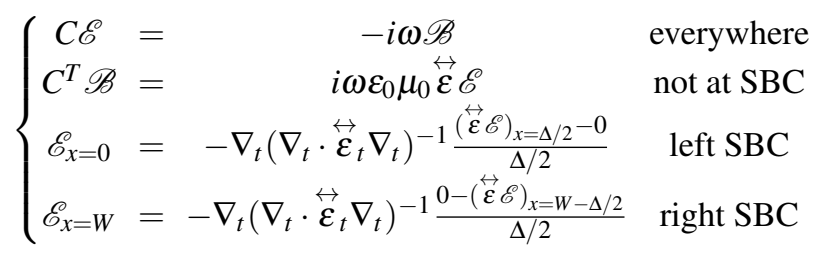

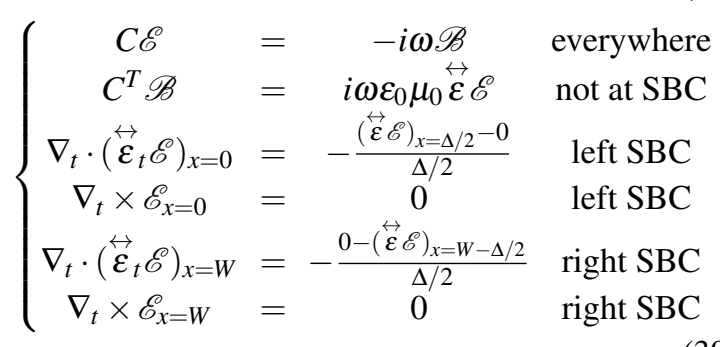

The minor additional difficulty here lies in the construction of discrete versions of $\stackrel{\leftrightarrow}{\varepsilon}$ and $\stackrel{\leftrightarrow}{\varepsilon}_{t}$. On a Yee grid, this is not entirely trivial ${ }^{14,16,25}$ unless $\overleftrightarrow{\varepsilon}$ is diagonal: in general, calculating $\vec{D}$ from $\vec{E}$ requires knowledge of all three components of $\vec{E}$ at the same point. Some interpolation as shown in figure 7 is therefore necessary.

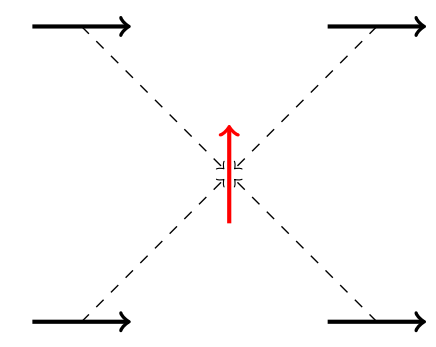

FIG. 7. Shown are 5 discretized electric field components on a slice of the Yee grid. In order to calculate $\vec{D}_{y}=(\stackrel{\leftrightarrow}{\varepsilon} \vec{E})_{y}$, where $\stackrel{\leftrightarrow}{\varepsilon}$ is the $3 \times$ 3 dielectric tensor, at the desired point (red), it is necessary to know all 3 electric field components at that point, so some interpolation is needed (gray dashed).

\section{E. Non-asymptotic case}

After discretizing the normal derivative, eq. (30) becomes

$$
\nabla_{t} \cdot\left[\Psi_{R F} \rightarrow \vec{D}_{t}\right] \Psi_{R F}=\frac{D_{n}(x=\Delta / 2)-D_{n}(x=0)}{\Delta / 2}
$$

Using the non-asymptotic SBC,

$$
\nabla_{t} \cdot\left[\Psi_{R F} \rightarrow \vec{D}_{t}\right] \Psi_{R F}=\frac{D_{n}(x=\Delta / 2)+\frac{\varepsilon_{s h}}{\delta_{s h}} \Psi_{R F}}{\Delta / 2}
$$


Thus

$$
\begin{gathered}
\left(\nabla_{t} \cdot\left[\Psi_{R F} \rightarrow \vec{D}_{t}\right]-\frac{2 \varepsilon_{s h}}{\delta_{s h} \Delta}\right) \Psi_{R F}=\frac{D_{n}(x=\Delta / 2)}{\Delta / 2} \\
\Psi_{R F}=\left(\nabla_{t} \cdot\left[\Psi_{R F} \rightarrow \vec{D}_{t}\right]-\frac{2 \varepsilon_{s h}}{\delta_{s h} \Delta}\right)^{-1} \frac{\vec{D}_{n}(x=\Delta / 2)}{\Delta / 2} \\
\vec{E}_{t}=-\nabla_{t}\left(\nabla_{t} \cdot\left[\Psi_{R F} \rightarrow \vec{D}_{t}\right]-\frac{2 \varepsilon_{s h}}{\delta_{s h} \Delta}\right)^{-1} \frac{\vec{D}_{n}(x=\Delta / 2)}{\Delta / 2}
\end{gathered}
$$

Thus, analogous to eq. (38), the discretized equations for the non-asymptotic case are

$$
\left\{\begin{array}{ccc}
C \mathscr{E} & = & -i \omega \mathscr{B} \\
C^{T} \mathscr{B} & = & i \omega \varepsilon_{0} \mu_{0} \stackrel{\leftrightarrow}{\mathscr{E}} \mathscr{E} \\
\mathscr{E}_{x=0} & = & -\nabla_{t}\left(\nabla_{t} \cdot\left[\Psi_{R F} \rightarrow \vec{D}_{t}\right]-\frac{2 \varepsilon_{s h}}{\delta_{s h} \Delta}\right)^{-1} \frac{(\overleftrightarrow{\varepsilon} \mathscr{E})_{x=\Delta / 2}-0}{\Delta / 2} \\
\mathscr{E}_{x=W} & =-\nabla_{t}\left(\nabla_{t} \cdot\left[\Psi_{R F} \rightarrow \vec{D}_{t}\right]-\frac{2 \varepsilon_{s h}}{\delta_{s h} \Delta}\right)^{-1} \frac{0-(\overleftrightarrow{\varepsilon} \mathscr{E})_{x=W-\Delta / 2}}{\Delta / 2}
\end{array}\right.
$$

\section{AN EXACT VACUUM SOLUTION TO VALIDATE THE NUMERICAL APPROACH}

Let us consider the vacuum case, where exact solutions of Maxwell's equations are most easily constructed.

The vacuum dispersion relation is $k^{2}=k_{x}^{2}+k_{y}^{2}+k_{z}^{2}=\frac{\omega^{2}}{c^{2}}$. Let us assume the fields vary either as $\exp \left(i\left(k_{y} y+k_{z} z+\right.\right.$ $\omega t)) \cos \left(k_{x} x\right)$ or as $\exp \left(i\left(k_{y} y+k_{z} z+\omega t\right)\right) \sin \left(k_{x} x\right)$, and let the sheath boundary condition $\left(\nabla_{t} \times \vec{E}=0, \vec{D}_{n}=0 \Longleftrightarrow \vec{E}_{n}=0\right)$ be at $x=0$.

We expect the electric field to be of the form

$$
\begin{aligned}
\frac{\vec{E}}{e^{i \omega t}} & =\eta_{1} e^{i\left(k_{y} y+k_{z} z\right)} \overrightarrow{1_{x}} \sin \left(k_{x} x\right) \\
& +\eta_{2}\left(\nabla_{t} \times e^{i\left(k_{y} y+k_{z} z\right)} \overrightarrow{1}_{x}\right) \sin \left(k_{x} x\right) \\
& +\eta_{3}\left(\nabla_{t} e^{i\left(k_{y} y+k_{z} z\right)}\right) \cos \left(k_{x} x\right)
\end{aligned}
$$

Note that the second and third terms are the Helmholtz decomposition of the 2D vector field given by the $y$ and $z$ components of $\vec{E}$ : only the curl-free part (the third term) is nonzero at $x=0$, which immediately implies that eq. (46) obeys the asymptotic sheath boundary condition by construction. All we have to do is find $\eta_{1}, \eta_{2}, \eta_{3}$ based on the wave equation

$$
\vec{k} \times(\vec{k} \times \vec{E})-\frac{\omega^{2}}{c^{2}} \vec{E}=0
$$

We find

$$
\begin{aligned}
\frac{\vec{E}}{e^{i \omega t}} & =\alpha_{1} e^{i\left(k_{y} y+k_{z} z\right)} \overrightarrow{1}_{x} \sin \left(k_{x} x\right) \\
& +\alpha_{2} \frac{c}{\omega}\left(\nabla_{t} \times e^{i\left(k_{y} y+k_{z} z\right)} \overrightarrow{1}_{x}\right) \sin \left(k_{x} x\right) \\
& +\alpha_{1} \frac{k_{x}}{k_{y}^{2}+k_{z}^{2}}\left(\nabla_{t} e^{i\left(k_{y} y+k_{z} z\right)}\right) \cos \left(k_{x} x\right)
\end{aligned}
$$

where $\alpha_{1}, \alpha_{2}$ are amplitudes for the two linearly independent solutions, with units of electric field strength $(\mathrm{V} / \mathrm{m}) . \overrightarrow{1_{x}}$ is the unit vector in the $x$ direction.

The RF potential is readily apparent from eq. (48):

$$
\Psi_{R F}=-\alpha_{1} \frac{k_{x}}{k_{y}^{2}+k_{z}^{2}} e^{i\left(k_{y} y+k_{z} z\right)}+\Psi_{0}
$$

where $\Psi_{0}$ is an arbitrary constant, which we will take to be 0 in the rest of this paper. Note that eq. (49) obeys eq. (23.a):

$$
\begin{aligned}
\nabla^{2} \Psi_{R F} & =-\nabla^{2} \alpha_{1} \frac{k_{x}}{k_{y}^{2}+k_{z}^{2}} e^{i\left(k_{y} y+k_{z} z\right)} \\
& =-\left(-k_{y}^{2}-k_{z}^{2}\right) \alpha_{1} \frac{k_{x}}{k_{y}^{2}+k_{z}^{2}} e^{i\left(k_{y} y+k_{z} z\right)} \\
& =\alpha_{1} k_{x} e^{i\left(k_{y} y+k_{z} z\right)} \\
& =\left.\alpha_{1} e^{i\left(k_{y} y+k_{z} z\right)} \partial_{x} \sin \left(k_{x} x\right)\right|_{x=0}
\end{aligned}
$$

\section{RESULTS}

\section{A. In vacuum}

The problem parameters are as follows: $W=1 \mathrm{~m}, H=$ $1 \mathrm{~m}, D=0.5 \mathrm{~m}, k_{x}=3 \pi / W, k_{z}=3 \pi / H, f=36.5 \mathrm{MHz}$. The number of Yee cells is $30 \times 20 \times 30$. The Yee cells thus have size $(1 / 30) \mathrm{m} \times(1 / 40) \mathrm{m} \times(1 / 30) \mathrm{m}$, more than enough to resolve the vacuum wavelength of $\lambda=8.2 \mathrm{~m}$, as well as the wavelengths in the $x$ - and $z$-directions $2 \pi / k_{x}=\frac{2}{3} W$ and $2 \pi / k_{z}=\frac{2}{3} H$, and the evanescence length in the $y$-direction $1 /\left|k_{y}\right|=7.5 \mathrm{~cm}$.

The source at $y=0$ is

$$
\begin{aligned}
\vec{E}_{t} & =-41.6 \sin \left(k_{x} x\right) \sin \left(k_{z} z\right) \overrightarrow{1}_{x} \\
& +\left(41.9 \cos \left(k_{x} x\right)+0.28 i \sin \left(k_{x} x\right)\right) \cos \left(k_{z} z\right) \overrightarrow{1}_{z}
\end{aligned}
$$

\section{Comparison with exact solutions}

We start from the exact solution eq. (48). To obey the PEC boundary conditions at $z=0, z=H$, we pick a linear combination of the upward-propagating and downward-propagating $\left(k_{z}= \pm 3 \pi / H\right)$ versions of eq. (48), such that $E_{x}, E_{y}$ become proportional to $\sin \left(k_{z} z\right)$ rather than $\exp \left(i k_{z} z\right)$.

In figures 8,9 and 10 , we show the numerical results obtained with the numerical scheme of section V C (specifically eq. (36)) and compare them with this exact result. We see that both components of the tangential electric field, and the RF potential $\Psi_{R F}$, are calculated correctly by this numerical scheme.

We do not plot $E_{x}$, the normal component. In the Yee cells, there are no degrees of freedom associated with $E_{x}$ on the surface, so plotting $E_{x}$ on the surface would amount to plotting 0 , and tell us nothing about the correctness of this scheme. 


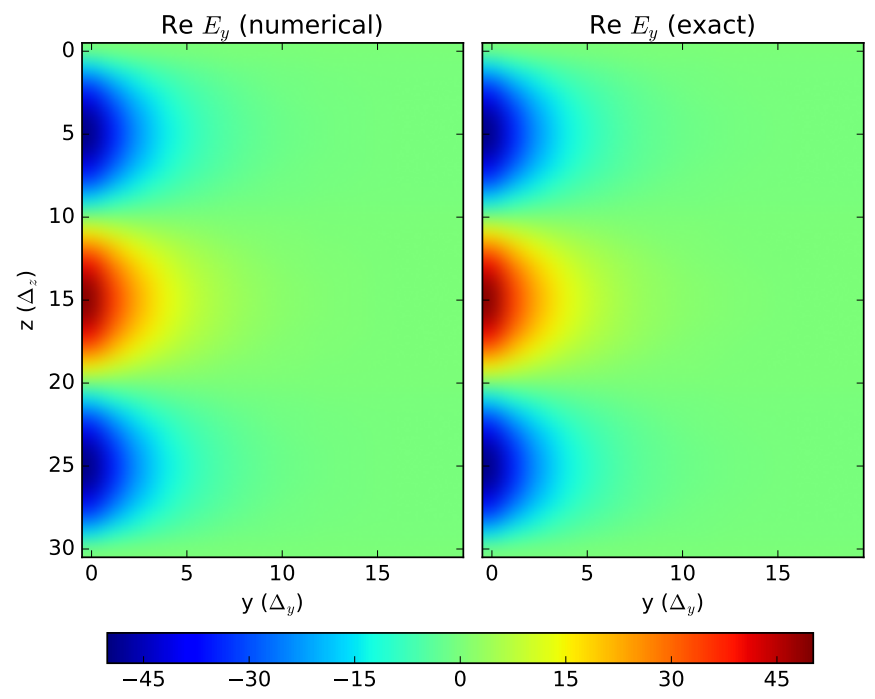

FIG. 8. Tangential $E_{y}$ field at the $x=0$ plane where a SBC is applied. Left: numerical, right: exact.

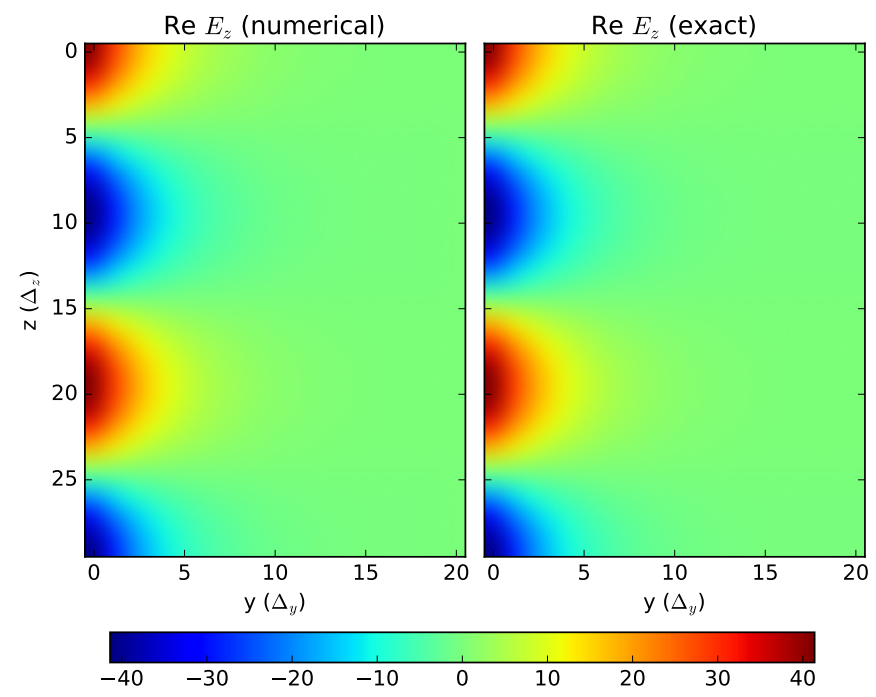

FIG. 9. Tangential $E_{z}$ field at the $x=0$ plane where a SBC is applied. Left: numerical, right: exact.

\section{Equivalence of eqs. (36) and (37)}

In sections V C and V D, we derived equivalent "Poisson" and "sparse"/"local" formulations. The "Poisson" formulations should be solved iteratively (we use python's gmres solver ${ }^{26}$ ), and require the solution of a Poisson-like equation at every iteration. Figures 11 and 12 demonstrate that both formulations give the same $\Psi_{R F}$.

To solve the Poisson-like equation at every iteration, we use a direct solver (figure 13). The size of the system of equations to be solved for the Poisson-like equations on the sheath surfaces is proportional to the sheath's surface area, and is only a tiny fraction of the total number of degrees of freedom in 3D. In figure 14, we compare the convergence of the Poisson approach with that of the sparse approach. We see that

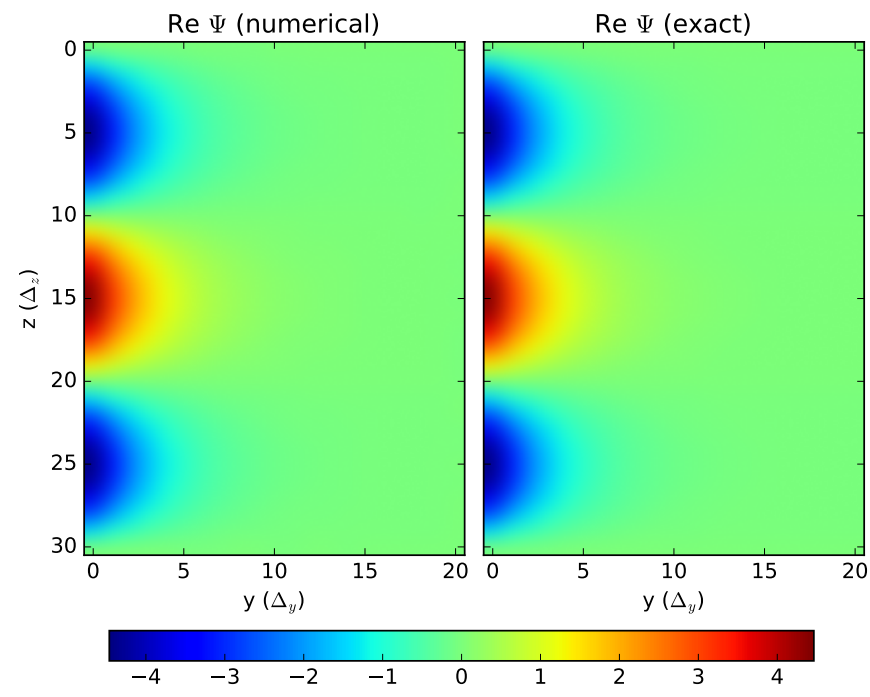

FIG. 10. RF potential $\Psi_{R F}$ at the $x=0$ plane where a SBC is applied. Left: numerical, right: exact. The tangential components in figures 8 and 9 are the negative gradient of this potential. Note that the very existence of this potential is only guaranteed thanks to the SBC.

the Poisson approach converges much faster. The sparse approach does not converge within a reasonable number of iterations, and requires a direct solver, which can rapidly become very memory demanding.

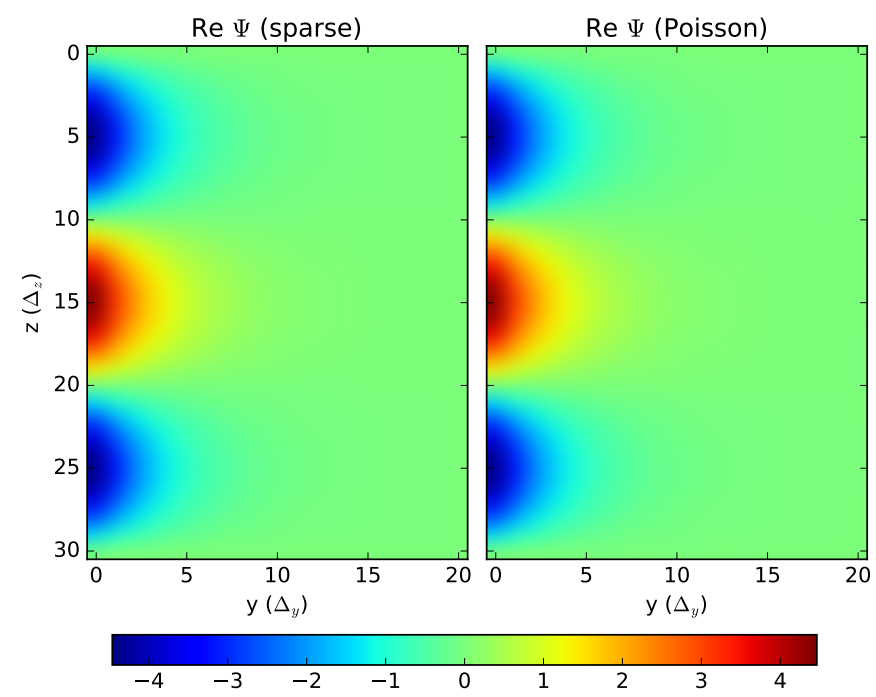

FIG. 11. Left: RF potential $\Psi_{R F}$ in vacuum as calculated numerically using the sparse formulation eq. (37) and a direct solver. Right: the same, calculated with the Poisson formulation eq. (36) and an iterative solver.

\section{B. In plasma}

We use a very tenuous hydrogen plasma with $n_{e}=n_{p}=$ $1.0 \cdot 10^{13} \mathrm{~m}^{-3}$ and $B_{0}=2 \mathrm{~T}$ in the $x$-direction (perpendicular to the sheaths). Again $W=1 \mathrm{~m}, H=1 \mathrm{~m}, D=0.5 \mathrm{~m}, k_{x}=$ 


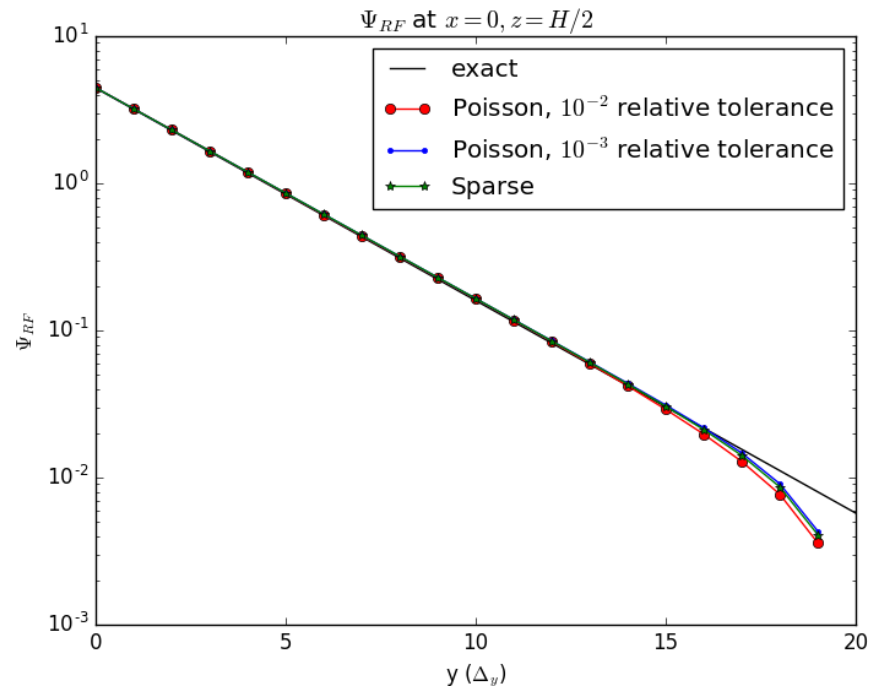

FIG. 12. 1D slice of figure 11 at $z=H / 2$. This again confirms the equivalence of the "Poisson" and "sparse" formulations. Note that the exact solution neglects reflections at $y=D=20 \Delta_{y}$, hence the slight deviation at high $y$.

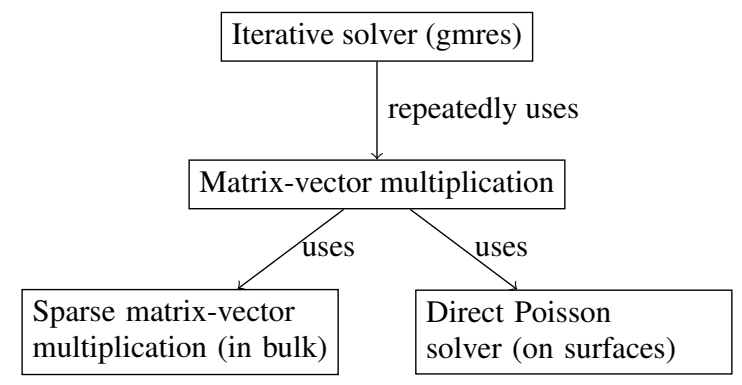

FIG. 13. An iterative solver for a linear system of equations $A x=b$ does not need an explicit expression for the matrix $A$, merely the ability to compute matrix-vector multiplications $A x$. In the Poisson approach, the matrix-vector multiplication makes use of a direct solver for the Poisson equations on the sheath surfaces.

$3 \pi / W, k_{z}=3 \pi / H, f=36.5 \mathrm{MHz}$. The corresponding dielectric tensor (see eq. (16)) is

$$
\stackrel{\leftrightarrow}{\varepsilon}=\left[\begin{array}{ccc}
0.395 & 0 & 0 \\
0 & 0.999 & -\left(13 \cdot 10^{-4}\right) i \\
0 & \left(13 \cdot 10^{-4}\right) i & 0.999
\end{array}\right]
$$

The number of Yee cells is $60 \times 20 \times 60$.

Corresponding with these values of $k_{x}, k_{z}, \omega$, there are four possible values for $k_{y}$, two for the fast wave and two for the slow wave, both of which are evanescent:

$$
\begin{aligned}
& k_{y, 1}^{2}=-92.5 \mathrm{~m}^{-2} \\
& k_{y, 2}^{2}=-98.1 \mathrm{~m}^{-2}
\end{aligned}
$$

We use a source of the form $E_{x}=\sin \left(k_{x} x\right) \sin \left(k_{z} z\right)$. This implies that the Dirichlet boundary condition for $\Psi_{R F}$ is that $\Psi_{R F}$ is constant on all four edges, and we arbitrarily chose this constant to be 0 . The resulting $\Psi_{R F}$ is shown in figure 15. It

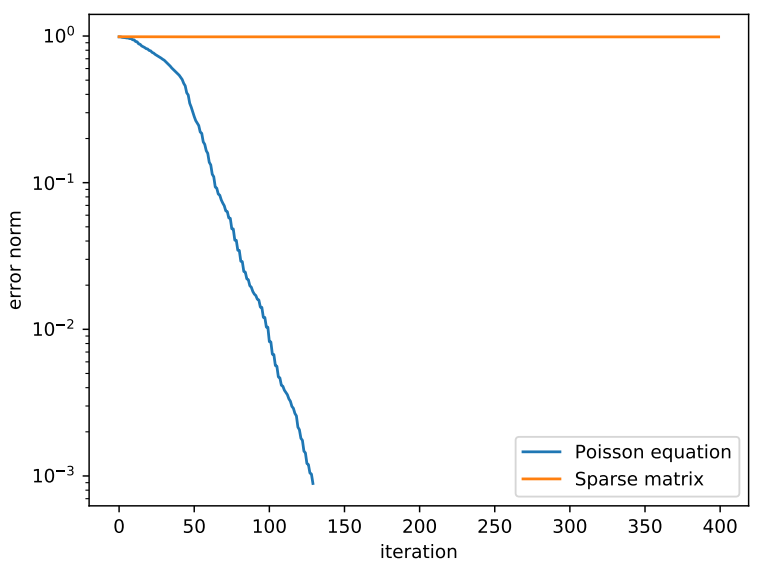

FIG. 14. Convergence using the gmres iterative solver, when solving eq. (36) (by solving a Poisson equation at every iteration of the solver), vs. eq. (37) (by solving a purely sparse system of equations). The sparse approach eq. (37) converges either very slowly or not at all, we must use a direct solver if we want to use it.

looks like

$$
\Psi_{R F} \propto \sin \left(k_{z} z\right)\left(\exp \left(-\left|k_{y, 1}\right| y\right)-\exp \left(-\left|k_{y, 2}\right| y\right)\right)
$$

which is what we should expect: this is the only linear combination of complex exponentials with the expected wavenumbers that obeys the Dirichlet boundary conditions.

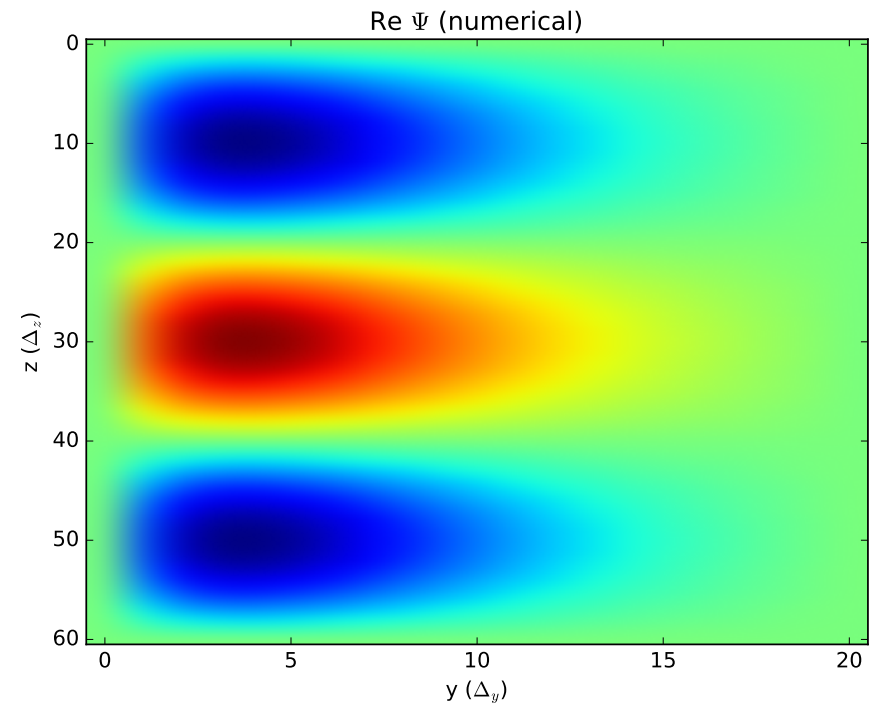

FIG. 15. RF potential $\Psi_{R F}$ in plasma with dielectric tensor given by eq. (52), with asymptotic sheath boundary conditions, as calculated numerically using eq. (38).

A denser and more tokamak-relevant plasma, with $n_{e}=$ $n_{p}=1.0 \cdot 10^{16} \mathrm{~m}^{-3}$ and other parameters the same as before, 
with dielectric tensor

$$
\overleftrightarrow{\varepsilon}=\left[\begin{array}{ccc}
-604 & 0 & 0 \\
0 & -0.09 & -1.31 i \\
0 & 1.31 i & -0.09
\end{array}\right]
$$

has fast and slow waves with evanescence lengths $\frac{1}{\left|k_{y}\right|}=7 \mathrm{~cm}$ and $\frac{1}{\left|k_{y}\right|}=0.1 \mathrm{~cm}$ respectively. We chose a much smaller $\Delta_{y}=$ $4 \cdot 10^{-4} \mathrm{~m}$ to (barely) resolve the evanescence length of the slow wave, and also reduce the vertical range to just half a vertical wavelength $\frac{\pi}{k_{z}}$. The numerically determined $\Psi_{R F}$ is shown in figure 16, and, like figure 15, it appears to behave like eq. (55).

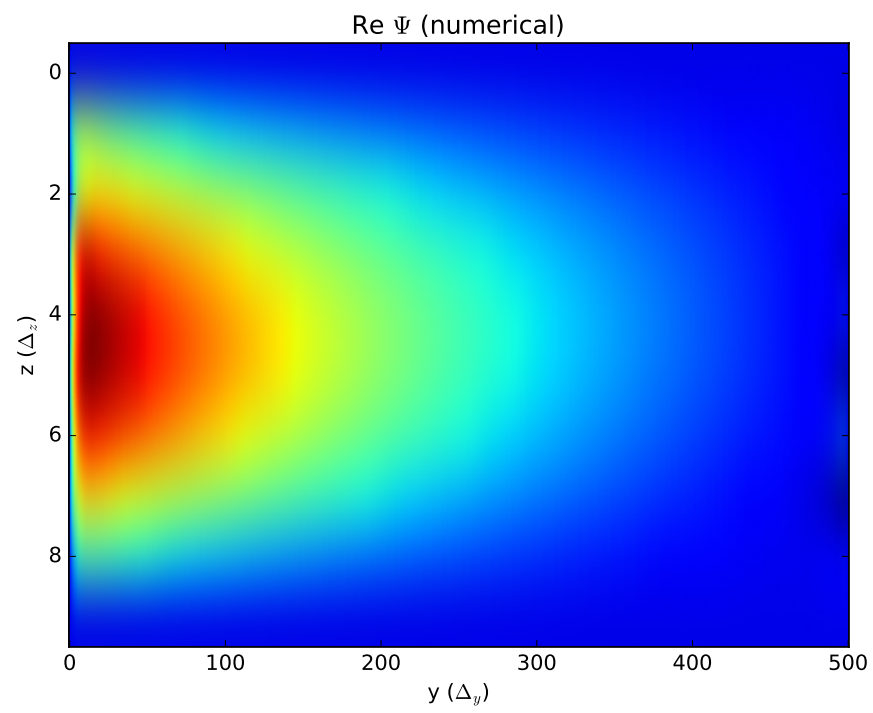

FIG. 16. RF potential $\Psi_{R F}$ in plasma with dielectric tensor given by eq. (56), with asymptotic sheath boundary conditions, as calculated numerically using eq. (38).

\section{CONCLUSION}

In this paper, we discussed boundary conditions of Maxwell's equations, both in vacuum and in cold magnetized plasma, which guarantee that the electric fields tangential to certain surfaces are conservative, even at nonzero frequency. Thus, a potential can be associated with these tangential fields, which is of great interest to sheath physics. We constructed and validated a numerical scheme by which Maxwell's equations (in cold plasma in frequency domain) can be solved with these boundary conditions. This scheme requires the solution of a Poisson-like equation on the sheath surfaces at every iteration of an iterative solver, in order to constrain the electric field components tangential to the surface.

We have given numerical examples in a simple cuboid with a Yee cell discretisation, but that is for simplicity of implementation rather than being a real constraint: the Poissonlike equations that define the radiofrequency potential on the sheath surface, and thereby the tangential electric fields (eqs. (23.a),(27) and (30) for the vacuum, plasma, and nonasymptotic cases respectively), can easily be discretized on any surface with any type of polygonal mesh ${ }^{24,27}$.

Although the inability to solve Maxwell's equations with the sheath boundary conditions discussed here has been a reason why the SSWICH sheath-modelling code remains 2D, it is not the only reason: there is also the need to resolve characteristic length scales of both the fast wave and the slow wave. These length scales typically differ by a factor of the order $\sqrt{m_{e} / m_{i}}$, and resolving both may require a very dense mesh $^{28}$, an issue already encountered in our numerical examples. In some realistic cases, a mesh density may be required that is denser than what is feasible with available computational resources.

In future work, we intend to implement this technique in a Finite Element solver ${ }^{11,12,19,20}$, which will enable greater geometrical flexibility than the Finite Difference approach discussed here. We will integrate it within the wider SSWICH framework (section II) to enable true 3D calculation of the DC sheath potential.

\section{ACKNOWLEDGMENTS}

This work has been carried out within the framework of the EUROfusion Consortium and has received funding from the Euratom research and training programme 2014-2018 and 2019-2020 under grant agreement No 633053. The views and opinions expressed herein do not necessarily reflect those of the European Commission.

${ }^{1} \mathrm{~J}$. Jacquot, Description non linéaire auto-cohérente de la propagation d'ondes radiofréquences et de la périphérie d'un plasma magnétisé, $\mathrm{Ph} . \mathrm{D}$. thesis, Université de Lorraine (2013).

${ }^{2}$ W. Tierens, J. Jacquot, V. Bobkov, J. Noterdaeme, L. Colas, and The AUG Team, "Nonlinear plasma sheath potential in the ASDEX upgrade 3-strap antenna: a parameter scan," Nuclear Fusion 57, 116034 (2017).

${ }^{3} \mathrm{~L}$. Lu, Modelling of plasma-antenna coupling and non-linear radio frequency wave-plasma-wall interactions in the magnetized plasma device under ion cyclotron range of frequencies, Ph.D. thesis, Ghent University (2016).

${ }^{4}$ L. Colas, J. Jacquot, J. Hillairet, W. Helou, W. Tierens, S. Heuraux, E. Faudot, L. Lu, and G. Urbanczyk, "Perfectly matched layers for time-harmonic transverse electric wave propagation in cylindrical and toroidal gyrotropic media," Journal of Computational Physics (2019).

${ }^{5}$ J. R. Myra and D. A. D'Ippolito, "Radio frequency sheaths in an oblique magnetic field," Physics of Plasmas 22, 062507 (2015), https://doi.org/10.1063/1.4922848.

${ }^{6} \mathrm{H}$. Kohno and J. Myra, "A finite element procedure for radio-frequency sheath-plasma interactions based on a sheath impedance model," Computer Physics Communications 220, 129-142 (2017).

${ }^{7} \mathrm{H}$. Kohno and J. Myra, "Radio-frequency wave interactions with a plasma sheath in oblique-angle magnetic fields using a sheath impedance model," Physics of Plasmas 26, 022507 (2019).

${ }^{8}$ D. D'Ippolito, J. Myra, E. F. Jaeger, and L. A. Berry, "Far-field sheaths due to fast waves incident on material boundaries," Physics of Plasmas 15, 102501 (2008).

${ }^{9}$ L. Colas, J. Jacquot, S. Heuraux, E. Faudot, K. Crombé, V. Kyrytsya, J. Hillairet, and M. Goniche, "Self consistent radio-frequency wave propagation and peripheral direct current plasma biasing: Simplified three dimensional non-linear treatment in the "wide sheath" asymptotic regime," Physics of Plasmas 19, 092505 (2012).

${ }^{10}$ J. Jacquot, D. Milanesio, L. Colas, Y. Corre, M. Goniche, J. Gunn, S. Heuraux, and M. Kubič, "Radio-frequency sheaths physics: Experi- 
mental characterization on tore supra and related self-consistent modeling," Physics of Plasmas 21, 061509 (2014), https://doi.org/10.1063/1.4884778.

${ }^{11}$ W. Tierens, D. Milanesio, G. Urbanczyk, W. Helou, V. V. Bobkov, J.-M. Noterdaeme, L. Colas, and R. Maggiora, "Validation of the ICRF antenna coupling code RAPLICASOL against TOPICA and experiments," Nuclear Fusion (2018).

${ }^{12}$ W. Tierens, G. S. López, R. Otin, G. Urbanczyk, L. Colas, R. Bilato, V. Bobkov, and J. Noterdaeme, "Recent improvements to the ICRF antenna coupling code RAPLICASOL," in 23rd topical conference on radiofrequency power in plasmas (2019).

${ }^{13}$ D. N. Smithe, D. A. D'Ippolito, and J. R. Myra, "Quantitative modeling of ICRF antennas with integrated time domain RF sheath and plasma physics," AIP Conference Proceedings 1580, 89-96 (2014), https://aip.scitation.org/doi/pdf/10.1063/1.4864506.

${ }^{14}$ T. Jenkins and D. Smithe, "Benchmarking sheath subgrid boundary conditions for macroscopic-scale simulations," Plasma Sources Science and Technology 24, 015020 (2014).

${ }^{15}$ T. G. Jenkins and D. N. Smithe, "Time-domain modeling of RF antennas and plasma-surface interactions," in EPJ Web of Conferences, Vol. 157 (EDP Sciences, 2017) p. 03021.

${ }^{16}$ D. N. Smithe, "Finite-difference time-domain simulation of fusion plasmas at radiofrequency time scales," Physics of Plasmas 14, 056104 (2007).

${ }^{17} \mathrm{~W}$. Tierens, Finite element and finite difference based approaches for the time-domain simulation of plasma-wave interactions, Ph.D. thesis, Ghent University (2013).

${ }^{18} \mathrm{~W}$. Tierens and D. De Zutter, "Finite-temperature corrections to the timedomain equations of motion for perpendicular propagation in nonuniform magnetized plasmas," Physics of Plasmas 19, 112110 (2012).

${ }^{19}$ R. Otin, "ERMES: A nodal-based finite element code for electromag- netic simulations in frequency domain," Computer Physics Communications 184, 2588-2595 (2013).

${ }^{20}$ R. Otin, W. Tierens, F. Parra, S. Aria, E. Lerche, P. Jacquet, I. Monakhov, and P. Dumortier, "Full wave simulation of ICRF waves in cold plasma with the stabilized open-source finite element tool ERMES," in 23rd topical conference on radiofrequency power in plasmas (2019).

${ }^{21}$ P. Pucci and J. Serrin, "The strong maximum principle revisited," Journal of Differential Equations 196, 1 - 66 (2004).

${ }^{22} \mathrm{~K}$. Yee, "Numerical solution of initial boundary value problems involving maxwell's equations in isotropic media," IEEE Transactions on antennas and propagation 14, 302-307 (1966).

${ }^{23}$ J. R. Nagel, "Numerical solutions to poisson equations using the finitedifference method [education column]," IEEE Antennas and Propagation Magazine 56, 209-224 (2014).

${ }^{24}$ J. Whiteman, "Lagrangian finite element and finite difference methods for poisson problems," in Numerische Behandlung von Differentialgleichungen (Springer, 1975) pp. 331-355.

${ }^{25}$ W. Tierens and D. De Zutter, "An unconditionally stable time-domain discretization on cartesian meshes for the simulation of nonuniform magnetized cold plasma," Journal of Computational Physics 231, 5144-5156 (2012).

${ }^{26}$ E. Jones, T. Oliphant, and P. Peterson, "SciPy: Open source scientific tools for Python,” (2001-), [Online; accessed 27 Feb 2019].

${ }^{27}$ N. Sukumar and A. Tabarraei, "Conforming polygonal finite elements," International Journal for Numerical Methods in Engineering 61, 2045-2066 (2004).

${ }^{28}$ M. Usoltceva, W. Tierens, M. Ochoukov, A. Kostic, K. Crombé, S. Heuraux, and J.-M. Noterdaeme, "Simulations of the ICRF slow wave in 3D in COMSOL," submitted to Plasma Physics and Controlled Fusion (2019). 